\title{
Clinical Sequelae of the Novel Coronavirus: Does COVID-19 Infection Predispose Patients to Cancer?
}

\author{
Priya Hays* \\ Principal, San Mateo, CA 94402, USA \\ ${ }^{\star}$ Corresponding author: Principal, San Mateo, CA 94402, USA; E-mail: priya.hays@outlook.com
}

Received: April 08, 2021; Accepted: April 10, 2021; Published: April 15, 2021

\begin{abstract}
As cancer patients are clinically known to be predisposed to COVID-19 infection, a corollary question of whether COVID-19 infection predisposes to cancer is explored. This article seeks to establish an association between novel coronavirus sequelae and cancer. A literature review on COVID-19 mechanisms of action, molecular responses it elicits upon infection and tumorigenesis pathways is conducted to establish this association. Major signaling pathways implicated in aberrant cellular growth are activated, the ensuing cytokine storm weakens the immune system response to tumors, and patients may develop cancer as a result of superimposed mutagenic and/or carcinogenic events. Future work needs to be performed to support this hypothesis, both in in vitro models and preclinical studies. COVID-19 patients may need to be monitored post-infection for developing cancer.
\end{abstract}

Keywords: Clinical sequelae, Consequences of infection, COVID-19, Global pandemic, Innate and adaptive immune response, Novel coronavirus, Oncology, SARS-CoV-2

The novel coronavirus, and the COVID-19 infection it causes, has led to a global pandemic. From its first cases in Wuhan, China in December 2019, it has spread to United Kingdom, Italy, Spain and now the USA, among other countries, with devastation. A robust scientific and medical literature emerged to provide information on patients vulnerable to succumbing to the infection. Clinicians warned that cancer patients were particularly susceptible to the novel coronavirus and they, along with their treating oncologists, should remain vigilant [1]. Lung cancer patients are especially forewarned and should be considered a priority group in terms of COVID-19 prevention. The protection provisions and control measures aiming to protect lung cancer patients from COVID-19 pose increasing concerns [1]. During the COVID-19 outbreak period, differential diagnosis for fever and respiratory symptoms for lung cancer patients receiving antitumor treatment should take place, in order to evaluate the risk of COVID-19 [1]. Lung cancer patients require meticulous attention in their clinical management in order to protect them from COVID-19 [1]. Additionally, the American Society of Clinical Oncology released a series of statements urging appropriate care for oncology patients at risk for viral infection, and to treat cases with caution (email communication).

This review considers a differently posed question: what are the clinical sequelae of patients who are infected with the novel coronavirus and what medical problems might they face in the future post-infection? These patients are now faced with pneumonia, tissue damage and even multiple organ failure. While these symptoms may lead to morbidity, there is an asymptomatic population who will recover that may possess more health conditions. Even those who have mildto-moderate clinical presentation may be at risk for other illnesses. The focus in this article is on oncology, and is timely since the virus mediates an immune response, weakens the immune system and causes inflammation, and genetically enters and lyses host cells, and releases its genome into the cytoplasm. According to a recent review, survivors of COVID-19 infection endure long-term complications, but they have yet to be elucidated, however global mortality rates range between 1 and 2\% [2]. While this currently remains the case, along with considering some of the future clinical consequences of being infected with SARS-CoV-2, this article calls for subsequent studies into what remains in store for this distinct health population especially with regards to oncology.

\section{COVID-19 Infection: Epidemiology \& Clinical Presentation}

As of 7 April 2021, worldwide there were more than 133 million confirmed cases of COVID-19 with a mortality of about $2,891,875$. In the USA, there were more than 31 million confirmed cases of COVID-19 and more than 570,000 deaths [3]. The complete clinical manifestation is not clear yet, as the reported symptoms range from mild-to-severe, and may result in the mortality just indicated [4]. Fever, cough, myalgia or fatigue, pneumonia, and complicated dyspnea are the most commonly reported symptoms; headache, diarrhea, hemoptysis, runny nose and phlegm- producing cough are less commonly reported [4]. Symptoms of SAR-CoV-2 infection range in severity, from asymptomatic to mild to exhibiting aggressive lung disease, which sometimes results in death, and is more frequent in middle-aged and elderly patients with co-morbidities including "chronic respiratory disease, cancer, tumor surgery, cirrhosis, hypertension, coronary heart disease, diabetes and Parkinson's disease." [4] Risk factors include being elderly, having poor immune function, having chronic co-morbidities, long-term usage of immunosuppressive agents and having a prior history of surgery before 
admission [4]. Fever, decrease in white blood cells, and abnormal chest $\mathrm{X}$-ray revealing pulmonary infiltration and lack of disease resolution post-antibiotic treatment are detailed in case definition guidelines [4]. Our understanding of co-morbidities and risk factors is evolving as clinical data emerges. A recent study in the Journal of the American Medical Association considered the clinical outcomes and presentation of hospitalized COVID-19 patients in the New York City region, and that hypertension, diabetes and obesity were the most prevalent comorbidities. 2634 patients were either discharged or died, and within this group, ICU treatment (14.2\%), invasive mechanical ventilation (12.2\%) and kidney replacement therapy (3.2\%) were major interventions and ultimately $21 \%$ of patients died [5].

Additionally, heart damage, neurologic symptoms, kidney damage and blood clots have been observed in COVID-19 patients [6]. In China, it was observed that $40 \%$ of patients who remained seriously ill as a result of COVID-19 infection presented with arrhythmias, while another $20 \%$ harbored other types of cardiac dysfunctions [7]. In comparison to $4.5 \%$ of patients who recovered from infection and experienced no cardiac injury, 51\% who exhibited cardiac damage died [7]. Additionally it was observed by Chinese clinicians in another study that approximately one-third of the 214 hospitalized patients exhibited neurological symptoms, experienced mainly as headaches, dizziness, and loss of taste and smell [8]. Seizures and strokes were also reported, prompting clinical guidance to perform neurological exams on COVID-19 infected patients [6]. Loss of normal kidney function requiring dialysis occurred in $14-30 \%$ of ICU patients, reported in one study, while another found acute kidney injuries and presentation of the virus in the kidneys in 9 of 26 patients who died of infection [9]. Pulmonary embolism as a result of COVID-19 infection was reported to have higher incidence among patients who died of the disease when compared to survivors. [6]. A study of 81 patients residing in Wuhan found that 20 of them exhibited pulmonary embolism accompanied by death in eight of them. [10]. "Clinicians and researchers have yet to determine whether the new coronavirus is directly attacking those organs, or whether the injuries are caused by the patients' immune responses to infection. Additionally, there is variation in recovery in patients and evidence of long-term persistence of the virus that may be the etiology of lung inflammation and pneumonitis, and instances of hypoxia" [5]. Patients may be predisposed to cancer as a result of the organ damage the virus is associated with.

Studies have shown that post-viral infection is associated with neuropsychological and mental health sequelae, including depression and anxiety that have been speculated as a result of viral impact on the immune system, manifesting mainly as a viral-induced cytokine storm [11]. Viral invasion of the central nervous system, including dysfunction of the cytokine network, may lead to encephalitis, or acute seizures [11]. The Centers for Disease Controls reports that serious complications are associated with patients post infection, including myocardial inflammation, pulmonary function abnormalities and olfactory and gustatory dysfunction [12]. Long-term COVID-19 sequelae have also been observed in healthcare workers with viral reinfection, including peripheral neuropathy and autonomic instability associated with overwhelming fatigue, compelling specific return-to work guidance for healthcare personnel [13]. A recent study characterized COVID-19 sequelae in into two categories: symptomatic abnormalities existing 4-12 weeks after infection, and those sequelae occurring beyond 12 weeks of original onset of the virus. Reports have observed that symptoms such as dyspnea, fatigue and PTSD have persisted in the patients with "long COVID-19," and are associated with distinct pathophysiology and immunological abnormalities. Imminent hematological sequelae included pulmonary embolism and stroke, with viral induced coagulopathy associated with a "hyperinflammatory and hypercoagulable state" [14].

Many of these reports are observational, and sequelae that are distinctly associated and implicated with signaling pathways turned on by the virus may not be elucidated. This paper serves to show a distinct association between the severe immunological outbreak that the virus induces and correlate it with cancer, through an understanding of these pathways and how they may potentially lead to cancer, or oncologic sequelae.

\section{Structure \& Pathophysiology of SARS-CoV-2}

As shown in Figure 1, the virus is surrounded by an envelope and houses positive stranded RNA "with a nucleocapsid. For addressing pathogenetic mechanisms of SARS-CoV-2, its viral structure and genome must be considered" [2]. As shown in Figure 2, the ssRNA houses the genomic structure of the virus, that is $30 \mathrm{~kb}$ in length, and is considered the largest of known human RNA viruses, and is accompanied by a 5 '-cap and 3 '-poly A tail. The polyprotein $1 \mathrm{a} / 1 \mathrm{~b}$ is produced in host cells from the RNA. Figure 2 additionally shows that the virus houses the replication-transcription complex in double-membrane vesicles derived from the subgenomic RNA [2]. "Open reading frames serve as templates for the production of the subgenomic mRNAs. In between these open reading frames, transcription termination occurs at transcription regulatory sequences [2]. At least six open reading frames are present, including a frameshift able to block host innate immune response. The viral envelope, a structural protein, confers viral pathogenicity since it promotes viral assembly and release. However, many of these features (e.g., those of nonstructural protein 2, and 11) have not yet been described." [2].

"The spike glycoproteins of $\mathrm{CoV}$ are composed of two subunits $\mathrm{S} 1$ and S2. The S2 protein is highly conserved and contains a fusion peptide, a transmembrane domain and a cytoplasmic domain [2]. The $S$ subunits consists of homotrimers that compose the spikes on the viral surface which can transduce through host receptors. Thus, it could be a target for antiviral (anti-S2) compounds. On the contrary, the spike receptorbinding domain presents only a $40 \%$ amino acid identity with other SARS-CoVs [2]. Other structural elements on which research must necessarily focus are the ORF3b that has no homology with that of SARS-CoVs and a secreted protein (encoded by ORF8), which is structurally different from those of SARS-CoV" [2].

The presence of mutations and additional strains were recently reported, and a spike mutation may have jumped to humans in late 2019, triggered by these mutations. "Researchers analyzed the transmembrane helical segments in the ORF1ab encoded 2 (nsp2) and nsp3 and found that position 723 presents a serine instead of a glycine residue, while the position 1010 is occupied by proline instead of isoleucine" [2]. The matter of viral mutations is key for explaining potential disease relapses. 


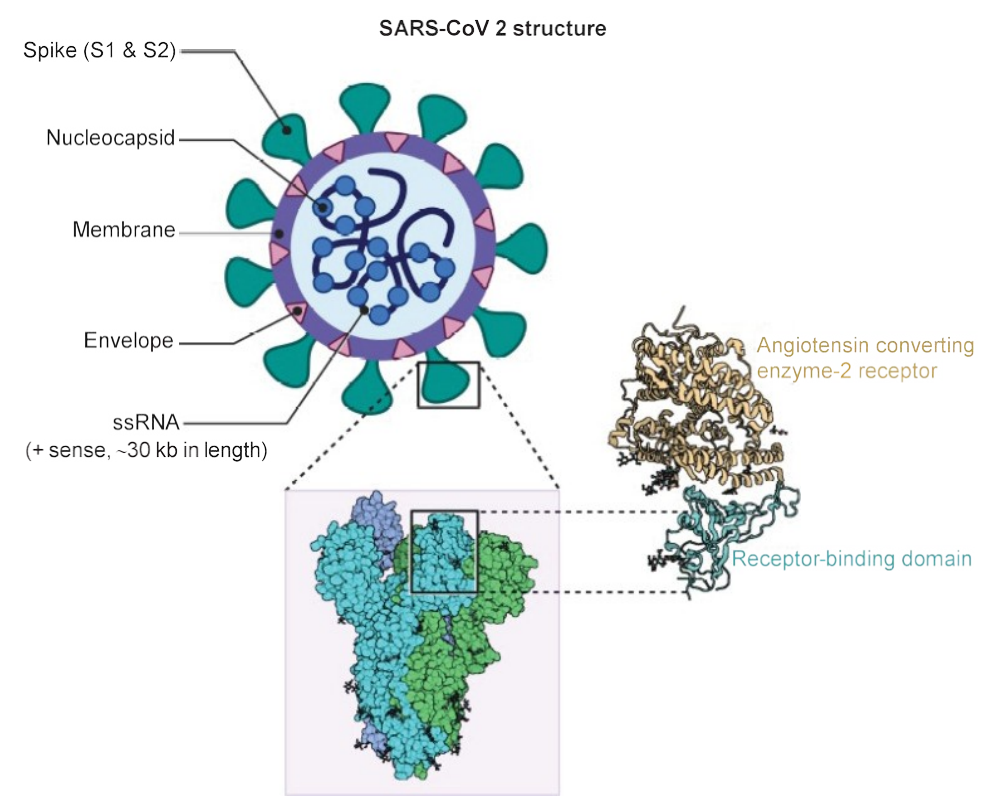

Figure 1: Structure of SARS-CoV2. A positive single-stranded RNA genome with a nucleocapsid is surrounded by a membrane with spike proteins. Reproduced with permission from [2] QC StatPearls (2020).

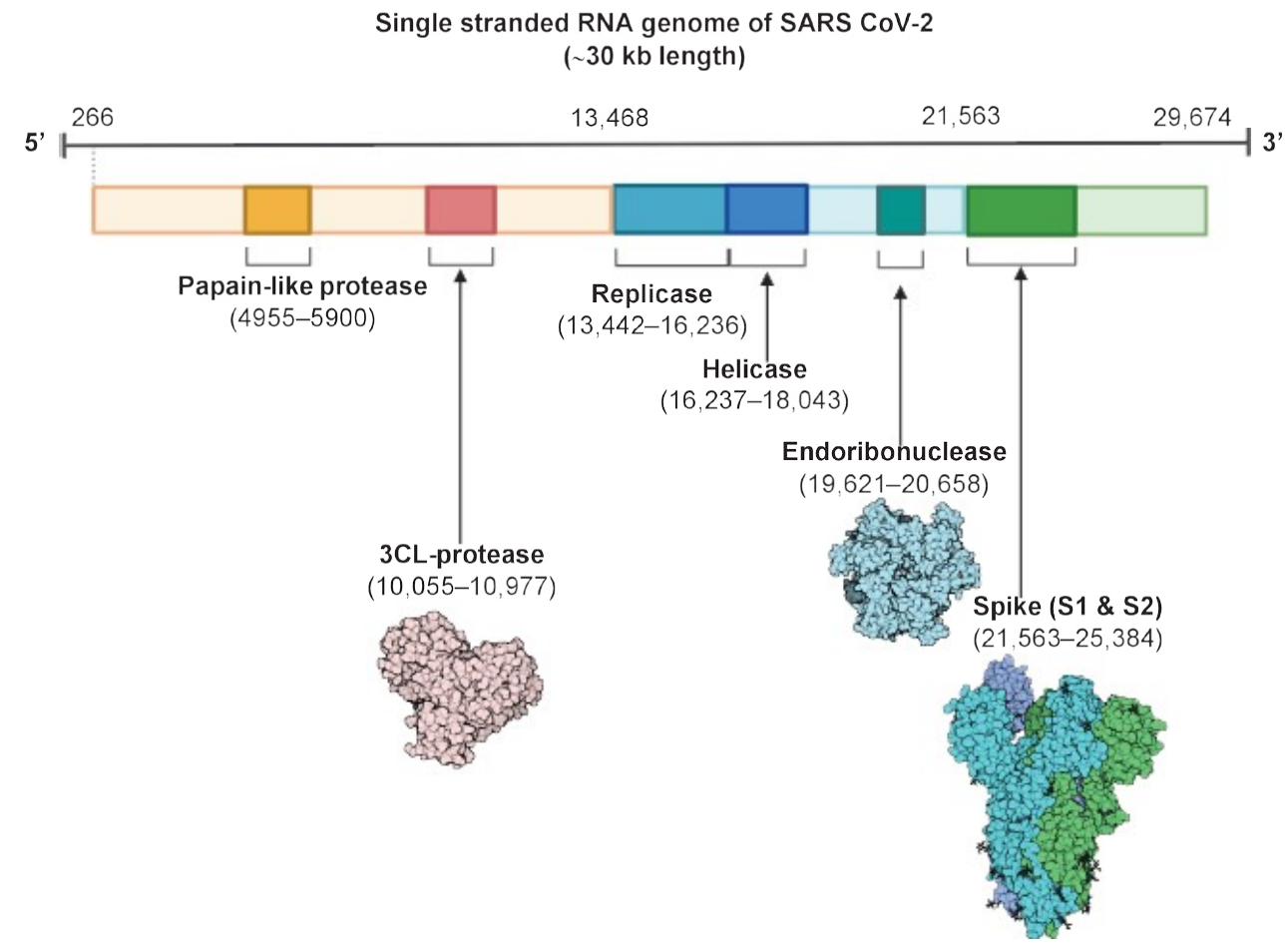

Figure 2: The SARS CoV-2 genomic structure. A papain-like protease, 3CL-protese and endoribonuclease are produced to lyse host cells. The replicase gene codes for replicative apparatus to create more virus to infect host cells. Reproduced with permission from [2], QC StatPearls (2020).

\section{COVID-19 Infection \& the Immune Response it Elicits}

\section{Immune Response against the Novel Coronavirus}

The basis for how a disrupted immune reaction leads to lung immunopathology resulting in adverse clinical outcomes after "pathogenic hCoV infection" was reported in prior studies. As early as 2017, Channappanavar and Perlman stated that the likelihood of the virus continuing to cross species barriers is high since SARS like coronovirus was identified in bats and MERS $\mathrm{CoV}$ was identified in domesticated camels. Thus there is higher probability of outbreaks occurring in humans [15]. A diverse variety of clinical presentation is observed in humans, with most patients exhibiting short periods of "moderate clinical illness" and a smaller but substantial fraction developing severe disease presenting with acute lung inflammation and acute respiratory disease [15]. 


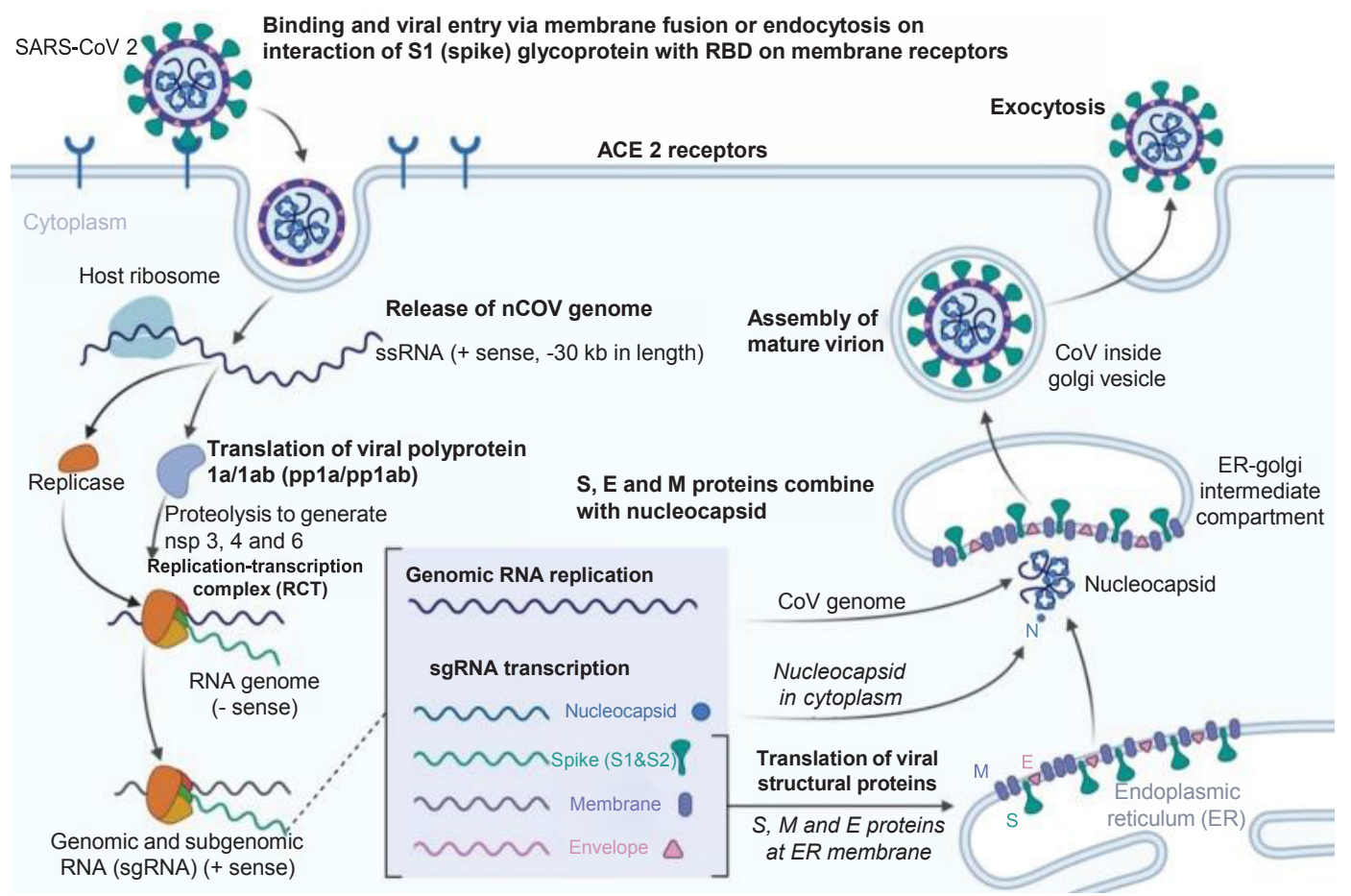

Figure 3: Coronavirus Infection Course. How the novel coronavirus infects host cells. E: Envelope; ER: Endoplasmic reticulum; M: Membrane; S: Spike. Reproduced with permission from [3].

There are certain inflammatory responses that may be conducive to cancer. IFN- $\alpha / \beta$ or inflammatory monocyte- macrophage-derived pro-inflammatory cytokines impede the clearance of the virus by sensitizing $\mathrm{T}$ cells to die and undergo apoptosis [15]. According to one recent report, "[t]he loss of TIR-domain-containing adapterinducing IFN- $\beta$ (TRIF), an adapter molecule for TLR-3 and TLR-4 signaling, resulted in a distinct inflammatory signature characterized by neutrophil and other inflammatory cell infiltration" [11]. "A dysregulated immune response to SARS-CoV in TRIF-deficient mice was associated with aberrant antiviral IFN (IFN- $\alpha$ and IFN- $\beta$ ), pro-inflammatory cytokine and chemokine (IL-6, TNF, IFN- $\gamma$ and monocyte chemoattractant CCL5), and interferon-stimulated gene (RSAD2, IFIT1 and CXCL10) responses", another possible indicator of cancer risk [15].

Interferon response is antagonized by the multiple proteins, both structural and nonstructural of SARS-CoV [5]. This antagonism may lead to delay in immune response or even evasion, which occurs in the early infection stage when the virus reaches high titer and inhibits interferon response as a result of viral sequestration of proteins [11]. In turn, apoptosis of T cells results, accompanied by a "dysfunctionally regulated inflammatory response" [15].

Some consequences of cytokine storm and immunopathology are epithelial and endothelial cell apoptosis and vascular leakage, suboptimal T-cell response, in other words. One of the key factors in viral clearance is $\mathrm{CoV}$ specific $\mathrm{T}$ cells that prevent more damage occurring to the host. These distinct $\mathrm{T}$ cell responses have a dampening effect on the overactive innate immune systemic response [15]. T cell response is mitigated as result of massive inflammatory effects resulting from pathogenic infection. T-cell death mediated by TNF leads to immunological infiltration by activated macrophages, aberrant homeostasis within tissues, "and acute respiratory distress syndrome." [15].

Figure 4 demonstrates the enormous immune response against the novel coronavirus [12]. The innate immune response and adaptive immunity have distinct responses to coronaviruses infection [16]. Macrophages are first infected by the $\mathrm{CoV}$ virus, which then present the viral antigens to $\mathrm{T}$ cells, which are then activated and further differentiate. Cytokines are then produced and released in a massive storm, leading to amplification of the immune response. In turn these mediators that are produced as a result of viral persistence mitigate the production of natural killer cells and activation of CD8+ T cells. This is a harmful outcome of viral proliferation since it is in fact these very same CD8+ $\mathrm{T}$ cells that are able to clear $\mathrm{CoV}$ through the production of effective mediators [16].

The release of genomic RNA into the cytoplasm occurs as a result of DPP4R attachment on the host cell, mediated by the S protein, leading to immunological response to the dsRNA generated during CoV replication. The dsRNA in turns sensitizes TLR-3 resulting in the activation of IRFs and NF- $\kappa B$ signaling pathways, which result in the production of "type I interferons and pro-inflammatory cytokines" Type I interferon is especially significant since it protects uninfected cells from "release of antiviral proteins." [16] "Sometimes, accessory proteins of $\mathrm{CoV}$ can interfere with TLR-3 signaling and bind the dsRNA of CoV during replication to prevent TLR-3 activation and evade the immune response." [16].

The MyD88-dependent signaling pathway is activated when TLR-4 binds to the $\mathrm{S}$ protein, thus causing the release of pro-inflammatory cytokines. Immune mediators are elicited in intense fashion due to 


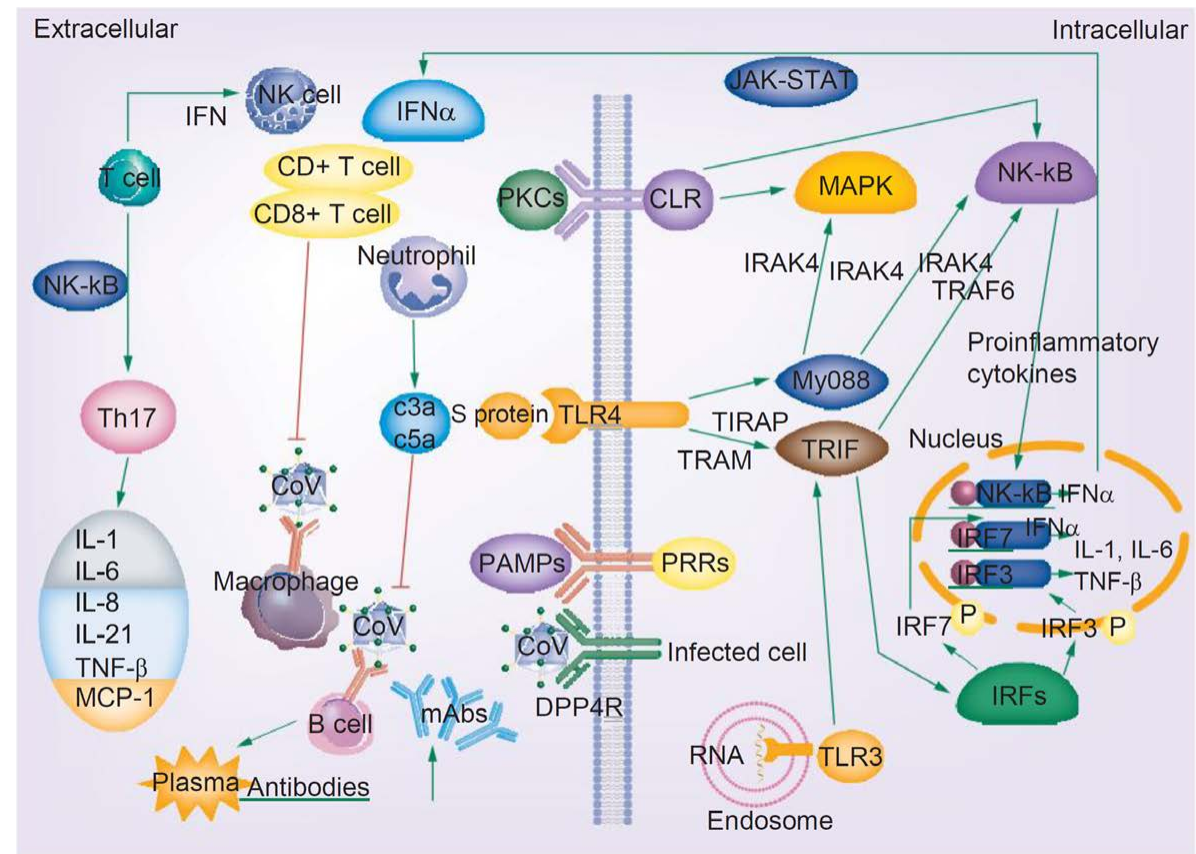

Figure 4: COVID-19 infection and the immune response. Activated signaling pathways such as JAK-STAT and MAPK,cytokine storm, T-cell depletion, humoral responses and high levels of inflammation may predispose patients infectedwith novel coronavirus to cancer. Reproduced with permission from [16].

viral-cell interactions. In short, a robust cytokine and chemokine response results in the form of secretion of IL-1, IL-6, IL-8, IL-21, TNF- $\beta$ and MCP-1, which in turn recruit more white blood cells to the infection site [16].

\section{Novel Coronavirus \& Oncologic Sequelae: How Cancer may be Implicated?}

PRRs (TLR, RIG-I-like receptor, NOD-like receptor, C-type lectin-like receptors and free-molecule receptors in the cytoplasm, such as cGAS, IFI16, STING and DAI) ensure that pathogen-associated molecular patterns (PAMPs) are recognized in various cellular compartments upon detection by the host innate immune system; PAMPs include bacterial and parasite lipoproteins and proteins. In turn additional pathogenic mechanisms are produced by different TLRs through the activation of adaptor proteins, (MyD88, TIRAP, TRIP and TRAM) that house similar TRR structure [16].

Viral infection plays a key role in signaling factor activation, that may have relevance for the initiation of cancer since a number of processes occur that lead to cellular growth. First, the adaptor protein MyD88, a member of the TIR family, "activates the transcription factors NF- $\mathrm{KB}$ and MAPKs pathways to induce inflammatory factors expression" and after activation, leads to the recruitment of receptorrelated kinases IRAK4, IRAKI, IRAK2 and IRAK-M [16]. Then, IRAK-TRAF6 interaction occurs, K-63 and NEMO ubiquitination takes place and serves as a further activator for NF- $\kappa$ B. In short, these TRIF-dependent activities lead to the activation of IRF3 and NF- $\kappa B$ as result of the recruitment of TRIF molecules to the TLR4 receptor, and the MyD88 adaptor protein to the TLR2 and TLR4 receptors, all happening through the function of TRAM and TIRAP [16].

Additionally, a "strong immune-mediated pneumonitis and delayed clearance of SARS-CoV from lungs" result as a result of depleted $\mathrm{CD} 4+\mathrm{T}$ cells through infiltration by white blood cells and cytokine production. The NF- $\kappa \mathrm{B}$ signaling pathway leads to the production of pro-inflammatory cytokines due to the increase in $\mathrm{T}$ helper cells. Other blood cells, such as monocytes and neutrophils, infiltrate the site of infection due to the production of IL-17 cytokine, inducing inflammation and activation of "other downstream cytokine and chemokine cascades, such as IL-1, IL-6, IL-8, IL-21, TNF- $\beta$ and MCP1 " [16].

There may be a distinct association between novel coronavirus infection and the onset of cancer through the activation of the MAPK and JAK-STAT signaling pathways and the NF- $\kappa B$ transcription factor. The MAPK signaling pathway, activated upon COVID-19 infection, is involved in the tumorigenesis of a number of cancers, including hepatocellular carcinoma, adrenocortical cancer, endometrial cancer, colorectal cancer and pituitary adenomas. 50-100\% of hepatocellular carcinomas display activation of the Ras/MAPK pathway, which is also associated with poor prognosis [17]. "The MAPK signaling pathway is also implicated in pituitary adenomas and because of the important roles of MAPK signaling pathways in tumorigenesis, the use of the MAPK signaling pathways as therapeutic targets has continuously been considered as a promising strategy for pituitary adenoma therapy." [18]. In the context of the role of two of the MAPK signaling pathways (ERKs $1 / 2$ and $\mathrm{p} 38$ ), adrenocortical tumor genesis is implicated by data suggesting that the MEK MAPK ERK signaling plays as a role, leading to potential clinical use as a diagnostic biomarker for malignancies and the development of targeted therapies [19]. Cholesterol in the form of LDL enhances intestinal inflammation and colorectal cancer progression via activation of reactive oxygen species (ROS). LDL cholesterol also has been shown to enhance intestinal inflammation leading to progression to colorectal cancer 
via activation of reactive oxygen species and the MAPK signaling pathway [20] Endometrial cancer is promoted through the activation of the MAPK signaling pathway by the estrogen receptor $(\boldsymbol{\alpha})$ [21]. The oncologic sequelae of COVID-19 may include these cancers upon viral activation of the MAPK signaling pathway. These tumorigenic pathways may play a role in cancer initiation and progression in COVID-19 infection.

The viral immune response is also implicated in other tumorigenic pathways, including those leading to lung cancer, breast cancer and T cell lymphomas. Type I interferon synthesis as a result of IRF3 and IRF7 activation, in turn activates the JAK-STAT signaling pathway involved in these tumorigenic processes, ultimately through PRRs that recognize viral RNA [22]. "JAK-STAT signaling pathways are mutationally activated in many extranodal T-cell lymphomas, such as natural killer/T-cell and hepatosplenic T-cell lymphomas." [23] "Since the JAK-STAT pathway is considered to be a central player in inflammation-mediated tumorigenesis, the implication of JAK-STAT signaling and the therapeutic potential of JAK1/2 inhibition was investigated in K-RAS-driven lung adenocarcinoma, and data showed that JAK1 and JAK2 are activated in human lung adenocarcinoma and that increased activation of JAK-STAT signaling correlated with disease progression and K-RAS activity in human lung adenocarcinoma" [24]. "Dysregulated JAK/STAT signaling has been implicated in breast cancer metastasis, which is associated with high relapse risks, which may be mediated through GRAM1b" [25].

NF- $\kappa B$, and TRIF-dependent pathways are also activated; and, IRF3 and IFN- $\beta$ are activated in turn [16]. Transcription factors IRF3 and NF- $\kappa B$ induce the gene expression of type I interferon through their activation by adaptor protein TRIF of TLRs. TLRs classify these types of signaling pathways induced by viral infection: "The TLR signaling pathways are classified as the MyD88-dependent pathway, which functions to activate immune inflammatory factors and the TRIF-dependent pathway, which then in turn functions to activate the type I interferons and inflammatory factors" [16]. NF-kB-driven gene products include cytokines/chemokines, growth factors, antiapoptotic factors, angiogenesis regulators and metalloproteinases, and drive oncogenesis. For instance, many of the genes transcribed by NF- $\kappa \mathrm{B}$ promote gastric carcinogenesis. Since it has been shown that chemotherapy-caused "cellular stress could elicit activation of the survival factor NF- $\kappa B$, which leads to acquisition of chemoresistance, the NF- $\kappa B$ system is recommended for therapeutic targeting" [26]. "Prosaposin, a neurotrophic factor, promotes the proliferation and tumorigenesis of glioma through TLR4-mediated NF- $\mathrm{KB}$ signaling pathways" [27].

Dendritic cells (DCs) also respond to the virus, and their differentiation from precursor cells to mature cells may be inhibited as is the case with HIV-1. If their maturation is blocked, by perhaps the lack of GM-CSF, IL- 4 and TNF- $\alpha$, the adaptive immune response, which plays a key role in the cancer-immunity cycle may be inhibited (Figure 5). DC's are the major antigen-presenting cells in the organism, and contribute both types of immunity by activating T cells and B cells. "Immature DCs have strong migration ability, and mature DCs can effectively activate $\mathrm{T}$ cells in the central link of start-up, regulation and maintenance of immune responses [16]. Thus, if the maturation process of DCs is blocked, it directly affects the initiation of subsequent adaptive immune responses" that may need to be mobilized in attacking cancer [16].

Other types of viruses have ostensible effects on adaptive immunity. DC precursor cells are unable to differentiate into mature DCs under the state of HIV-1 Nef protein infection when inducers are in

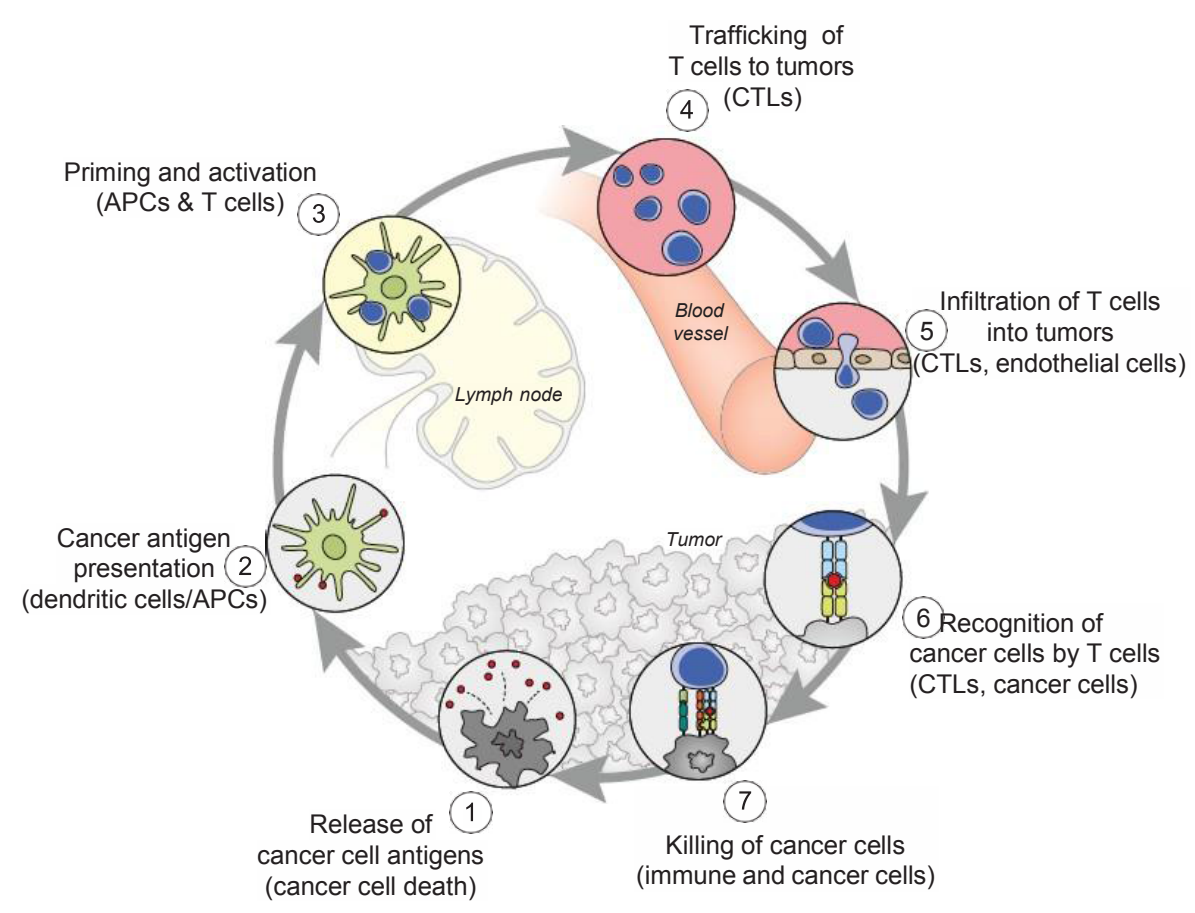

Figure 5: The cancer-immunity cycle. Dendritic cells present cancer antigens to $\mathrm{T}$ cell, which are honed to the cancer site. T cells infiltrate tumors and recognize cancer cells upon their destruction. Each point in this cycle may be points of vulnerability upon novel coronavirus infection. APC: Antigen-presenting cell. Reproduced with permission from [28]. 
the environment during HIV infection [16]. Thus, the Nef protein prevents the maturation of DCs from DC precursor cells [16]. Three molecules which exist on the surface of human peripheral blood mononuclear precursor cells (PBMCs), CD1a, CD1b, and DC-SIGN, are inhibited by the core protein and NS3 protein in HCV, and are central in the development of DCs from these PBMCs [16]. The viral antigen presentation process through DCs is reduced in HIV1 infection since MHC-I attentuation occurs on the DCs surface [16]. DC-SIGN expression, or DC specific intracellular adhesion molecule 3 grabbing nonintegrity, is facilitated by HIV-1 infection, and CC chemokine receptor 7 and MHC II molecules, two receptors which play key roles in the DC homing process, are inhibited. All of these processes accumulate to lead to an overall dysfunction of DCs, including their maturation and differentiation, hindering the crucial role they play in the adaptive immune response and allowing for successful viral evasion of the adaptive immune system [16]. If these DC events are occurring, cancer may co-opt normal cellular function and evade immune responses in a viral infection such as COVID-19.

A 'cytokine storm', an excessive immune reaction in the host, also results from viral mechanisms that results in extensive tissue damage, mediated by IL-produced by activated leukocytes. B lymphocyte differentiation, cell growth stimulation and pathogenesis of some types for cancer may result. These effects are linked to the function of structural and nonstructural proteins of the virus [2]. Cytokine release syndrome, an immune-related adverse effect of chimeric antigen receptor T-cell therapies, is also implicated in the potential emergence of cancer. Additionally, as tissue damage envelopes the body's physiological functioning, and cellular resources are increasingly devoted to compensating for it, cancer may emerge as a risk factor. Lung tissue damage, a prevalent and major consequence of infection, may be particularly at risk for developing into lung cancer, and patients who experience these symptoms, may need to be monitored for these sequelae.

\section{Relevance of the Cancer-immunity Cycle \& Tumor Microenvironment}

In the cancer-immunity cycle (Figure 5), the cancer presents antigens which antigen-presenting cells present to MHC to activate cytotoxic $\mathrm{CD}^{+} \mathrm{T}$ cells, to ultimately destroy cancer cells in a cyclic process [28]. In cancer, the cancer-immunity cycle is dysfunctional with the accumulation of inhibitors of T-cell response and promoters of cancer cell growth. In SARS-CoV infection, cytotoxic T cells kill virus, and lead to the production of antibodies specific to the virus through the activation of T dependent B cells. Further, lung inflammation leads to injury to the immune system as a result of activation of cytotoxic $\mathrm{T}$ cells. Immunosuppression, a risk factor for novel coronavirus, may emerge, conversely, as a risk for cancer in the context of viral infection.

From an understanding of the cancer-immunity cycle, are there are any elements of novel coronavirus infection that would make the cycle vulnerable to cancer at any point and prevent the generation of immunity to cancer? The presence of checkpoint inhibitors to immune responses, such as cytotoxic T-lymphocyte associated protein-4, programmed death-1 and programmed death-ligand-1, may inure the development of cancer with viral infection. In a depleted T-cell environment postinfection, the broad T-cell response to cancer may be undermined. This inflammatory response concomitant with immune cell depletion, whether temporary or not, may lead to oncologic sequelae if accompanied by a superimposed mutagenic and/or carcinogenic event potentially occurring in a time- adjacent manner. Figure 6 displays the tumor microenvironment in the context of breast cancer and illustrates major inflammation and cytokine release, marks of viral infection which may predispose to cancer.

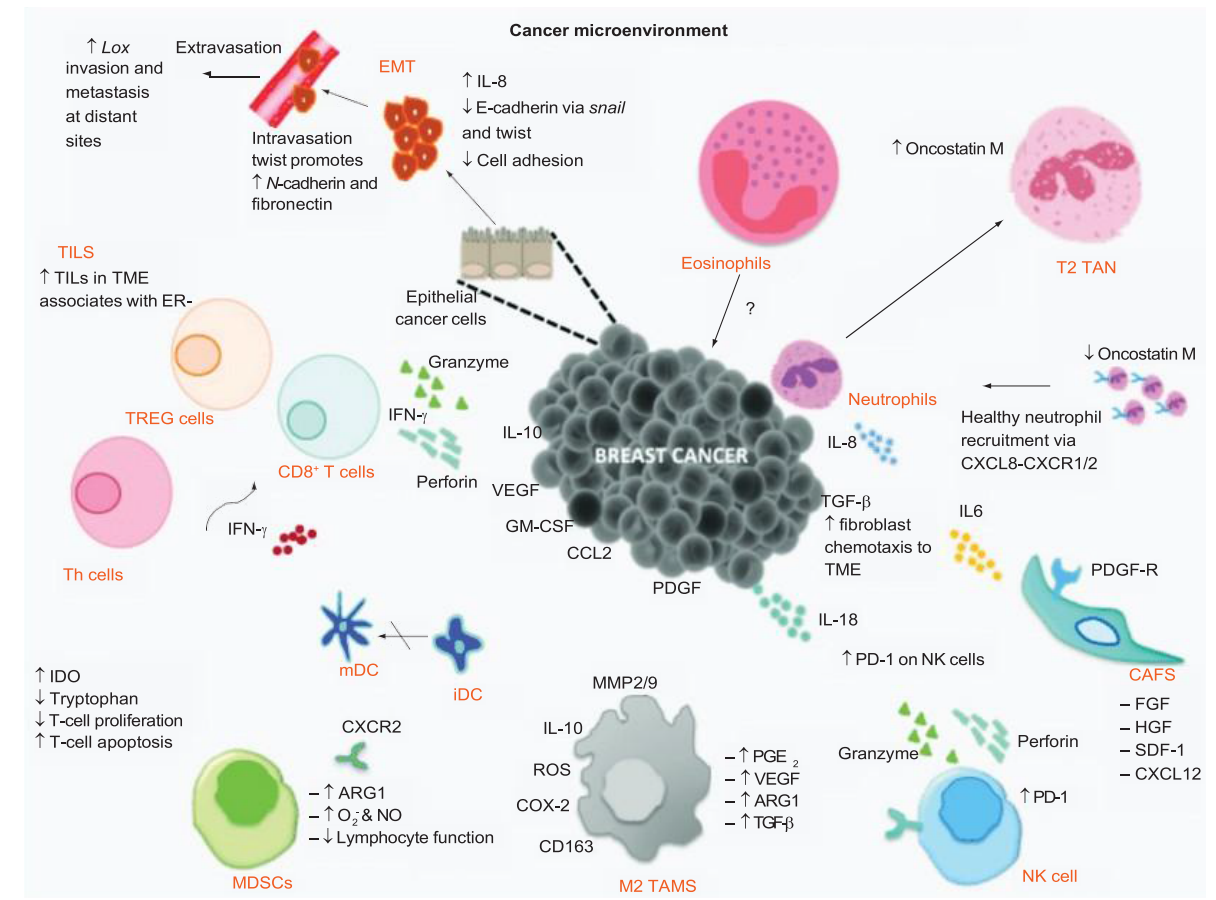

Figure 6: Tumor microenvironment of breast cancer. The tumor microenvironment progresses through cytokine release. Reproduced with permission from [29]. 


\section{Somatic Mutations, Drug Resistance \& COVID-19}

The implications for novel coronavirus infection on genomic variability are unknown at this point. If the virus through its replicative [30] and recombination capabilities [31] leads to genetic alterations that may predispose to cancer, another risk factor may be revealed.

The administration of antiviral agents and other proposed treatments such as the antimalarial drug hydroxy- chloroquine may induce drug resistance to cancer therapies, such as targeted agents and immunotherapies. Even further, the status of targeted therapies may be jeopardized by the infection of the virus, since many personalized agents target growth factors that are possibly affected by viral transduction; however, this remains to be investigated.

\section{Countervailing Viewpoints}

It is generally known that viruses can cause cancer by integration of the genomes into host cell chromosomes and the expression of oncoproteins. While both of these mechanisms are not implicated in SARS-CoV-2, these are not exclusive mechanisms. The turning on of oncogenic signaling pathways and the acute inflammatory response that results upon coronavirus infection can be hypothesized as being cancer inducing, or leading to the risk of developing cancer, especially if the patient has a superimposed mutagenic or carcinogenic event occurring concomitantly, even if the virus does not cause a chronic infection like viruses such as HCV, HCV and EBV. Additionally, there exist limited resources and research as a result of the pandemic that may damage the prognosis of cancer patients. Novel coronavirus can destroy T cells, but unlike HIV, does not lead to persistent infection of T cells. Data suggest however that there is a general depletion of $\mathrm{T}$ cells in the COVID-19 infection environment, and this may affect the healthy progression of the cancer-immunity cycle. Also, there exist gaps in current knowledge on the novel coronavirus' impact on the immune system during co-infection by other viruses, such as HCV, HIV and EBV. Finally, the overwhelming organ dysfunction that novel coronaviruses results in some cases should be further investigated, and as possibly having an association with cancer. These should remain areas of research for future study.

\section{Future Perspective}

As the population infected with the novel coronavirus grows, and the infection spreads, its clinical sequelae may pose an issue of concern for physicians, and oncologists in particular. Future studies should focus on the predisposition of these recovering patients for cancer, and if these patients need to be monitored for the disease. It is the cumulative effect of many distinguishable aspects of coronavirus infection that leads to the increased predisposition to cancer, which then warrants closer follow-up in the future. The organ damage, inflammatory response and signaling pathways activation that occur upon viral infection may sum to significantly increase patients' predisposition to cancer, and this may be compounded by a superimposed mutagenic or carcinogenic event. The oncology field may evolve by investigating the potential tumor microenvironment in the context of SARS-CoV-2 infection, and if the virus can be considered an etiological agent in the development of cancer. Clinical sequelae may involve physicians needing to refer COVID-19 cases to oncologists for the confirmation of cancer, either benign and malignant cases. The caveat must be mentioned however that knowledge surrounding coronavirus is constantly being updated and this review must be interpreted in light of any recently updated knowledge.

\section{Executive Summary}

- The novel coronavirus is leading to a global pandemic and is causing worldwide clinical and economic devastation. The infection may pose medical risk factors for patients, both symptomatic and asymptomatic, who recover. There will be a distinct health population in the future that will need to deal with clinical sequelae, and cancer may be one of them.

\section{Oncologic Sequelae of the Novel Coronavirus}

- Viral infection induces a robust immune response, a 'cytokine storm' leading to tissue damage and inflammation, which may predispose to cancer. Signaling factors, such as MAPK and JAKSTAT, promoted by viral infection may lead to aberrant cellular growth that marks cancer. The maturation of dendritic cells may be inhibited, as shown in patients infected with HIV-1.

\section{The Cancer-immunity Cycle \& Tumorigenesis}

- The cancer-immunity cycle may be impaired upon viral infection, since T-cell apoptosis also occurs as a result of viral infection, leading to a state of immunosuppression. If concomitant with a mutagenic or carcinogenic event, a cancerous state may result. The 'cytokine storm' poses a risk factor for cancer in the tumor microenvironment.

\section{Summary}

- In conclusion, patients infected and recovered from novel coronavirus, particularly those with lung tissue damage, may need to be monitored for cancer, among other diseases.

\section{References}

Papers of special note have been highlighted as: • of interest; •• of considerable interest

1. Xu Y, Liu H, Hu K, Wang M. (2020) Clinical management of lung cancer patients during the outbreak of 2019 novel coronavirus disease. Zhongguo Fe Ai Za Zhi 23(3), 136-141. [crossref]

2. Cascella M, Rajnik M, Cuomo A, Dulebohn SC, Di Napoli R. (2020) Features evaluation and treatment coronavirus (COVID-19). In: StatPearls. StatPearls Publishing, FL, USA. [crossref]

Excellent overview of clinical symptoms and progression of COVID-19 infection patients. Excellent graphics on coronavirus genome and structure.

1. Worldometers info/coronavirus/ Accessed 7 April 2021.

3. Adhikari SP, Meng S, Wu YJ et al (2020) Epidemiology, causes, clinical manifestation and diagnosis, prevention and control of coronavirus disease (COVID-19) during the early outbreak period: a scoping review. Infect Dis Poverty 9(1), 29. [crossref]

- Very informative review article encompasses the epidemiology and clinical presentation of the coronavirus disease.

4. Richardson S, Hirsch JS, Narasimhan M et al (2020) Presenting characteristics, comorbidities, and outcomes among 5700 patients hospitalized with COVID-19 in the New York City area. JAMA e206775 (Epub ahead of print). [crossref]

5. One of the first major studies conducted at a hotspot of the pandemic on characteristics of coronavirus patients and their comorbities. 
6. Advisory Board: it's not just lungs: COVID-19 may damage the heart, brain, and kidneys (2020). https://www.advisory.com/daily-briefing/2020/04/17/organ-damage.

7. Shi S, Qin M, Shen B. (2020) Association of cardiac injury with mortality in hospitalized patients with COVID-19 in Wuhan, China. JAMA Cardiol. doi:10.1001/ jamacardio.2020.0950 (Epub ahead of print). [crossref]

8. Mao L, Jin H, Wang M et al (2020) Neurologic manifestations of hospitalized patients with coronavirus disease 2019 in Wuhan, China. JAMA Neurol. e201127 doi:10.1001/ jamaneurol.2020.1127 (Epub ahead of print). [crossref]

9. Su H, Yang M, Wan C et al (2020) Renal histopathological analysis of 26 postmortem findings of patients with COVID-19 in China. Kidney Int. doi:10.1016/j. kint.2020.04.003 (Epub ahead of print). [crossref]

10. Cui S, Chen S, Li X, Liu S, Wang F. (2020) Prevalence of venous thromboembolism in patients with severe novel coronavirus pneumonia. J Thromb Haemost. doi:10.1111/ jth.14830 (Epub ahead of print). [crossref]

11. Troyer EA, Kohn JN, Hong S. (2020) Are we facing a crashing wave of neuropsychiatric sequelae of COVID-19? Neuropsychiatric symptoms and potential immunological mechanisms. Brain Behav Immub 87:34-39. [crossref]

12. https://www.cdc.gov/coronavirus/2019-ncov/hcp/clinical-care/late-sequelae.html Accessed 7 Apr 2021.

13. Praschan N, Josephy-Hernandez S, Kim DD, Kritzer MD, Mukerji S et al (2021) Implications of COVID-19 sequelae for health-care personne. The Lancet Respiratory Medicine. 9(3): 230-231. [crossref]

14. Nalbandian A, Sehgal K, Gupta A, Madhavan MV, McGroder C et al. (2021): Postacute COVID-19 syndrome. Nature Medicine.

15. Channappanavar R, Perlman S. (2017). Pathogenic human coronavirus infections: causes and consequences of cytokine storm and immunopathology. Semin Immunopathol 39(5), 529-539. [crossref]

- An early article on the molecular responses to coronavirus infection and possibility of pandemics.

16. Li G, Fan Y, Lai Y et al (2020) Coronavirus infections and immune responses. J Med Virol 92(4), 424-432. [crossref]

•-An excellent overview with graphics on the immune-mediated responses to coronavirus.

17. Delire B, Starkel P. (2015) The Ras/MAPK pathway and hepatocarcinoma: pathogenesis and therapeutic implications. Eur J Clin Invest 45(6), 609-623. [crossref]

18. Lu M, Wang Y, Zhan X. (2019) The MAPK pathway-based drug therapeutic targets in pituitary adenomas. Front Endocrinol 10, 419-430. [crossref]
19. Pereira SS, Monteiro MP, Costa MM et al (2019) MAPK/ERK pathway inhibition is a promising treatment target for adrenocortical tumors. J Cell Biochem 120, 894-906. [crossref]

20. Wang C, Peiwei L, Xuan J et al (2017). Cholesterol enhances colorectal cancer progression via ROS elevation and MAPK signaling pathway activation. Cell Physiol Biochem 42, 728-742. [crossref]

21. Liu A, Zhang D, Yang X, Song Y. (2019). Estrogen receptor alpha activates MAPK signaling pathway to promote the development of endometrial cancer. J Cell Biochem 120, 17593-17601. [crossref]

22. Scott L, Gandhi MK. (2015) Deregulated JAK/STAT signalling in lymphomagenesis, and its implications for the development of new targeted therapies. Blood Rev 29, 405-415.

23. van Arnam JS, Lim MS, Elenitoba-Johnson KSJ. (2018) Novel insights into the pathogenesis of T-cell lymphomas. Blood 24, 2320-2330. [crossref]

24. Mohrherr J, Haber M, Breitnencecker K et al (2019) JAK-STAT inhibition impairs K-RAS-driven lung adenocarcinoma progression. Int J Cancer 145, 3376-3388. [crossref]

25. Khanna P, Lee JS, Sereemaspun A et al (2018) GRAMD1B regulates cell migration in breast cancer cells through JAK/STAT and Akt signaling. Sci Rep 22, 1-10.

26. Sokolova O, Naumann M. (2017) NF- $\mathrm{kB}$ signaling in gastric cancer. Toxins (Basel) 9(4), E119. [crossref]

27. Jiang Y, Zhou J, Luo P et al (2018) Prosaposin promotes the proliferation and tumorigenesis of glioma through toll-like receptor 4 (TLR4)-mediated NF- $\mathrm{kB}$ signaling pathway. EbioMedicine 37, 78-90. [crossref]

28. Chen DS, Mellman I. (2013) Oncology meets immunology: the cancer-immunity cycle. Immunity 39(1), 1-10. [crossref]

- Seminal article on the cancer-immunity cycle, which may be implicated in novel coronavirus infection.

29. Barriga V, Kuol N, Nurgali K, Apostolopoulos V. (2019) The complex interaction between the tumor micro-environment and immune checkpoints in breast cancer. Cancers (Basel) 11(8), E1205. [crossref]

30. Chen Y, Liu Q, Guo D. (2020) Emerging coronaviruses: genome structure, replication and pathogenesis. J Med Virol 92, 418-423. [crossref]

31. Bentley K, Evans DJ. (2018) Mechanisms and consequences of positive-strand RNA virus recombination. J Gen Virol 99(10), 1345-1356. [crossref]

\section{Citation:}

Hays P (2021) Clinical Sequelae of the Novel Coronavirus: Does COVID-19 Infection Predispose Patients to Cancer? Prev Med Epid Public Heal Volume 2(2): 1-9. 